\title{
SUBRINGS OF I-RINGS AND S-RINGS
}

\author{
MAMADOU SANGHARE \\ Département de Mathématiques et Informatiques \\ Faculté des Sciences et Techniques \\ UCAD \\ DAKAR (SENEGAL) \\ e-mailsanghare@ucad.refer.sn
}

(Received May 6, 1993 and in revised form February 13, 1997)

\begin{abstract}
Let $R$ be a non-commutative associative ring with unity $1 \neq 0$, a left $R$-module is said to satisfy property (I) (resp. (S)) if every injective (resp. surjective) endomorphism of $M$ is an automorphism of $M$. It is well known that every Artinian (resp. Noetherian) module satisfies property (I) (resp. (S)) and that the converse is not true. A ring $R$ is called a left I-ring (resp. S-ring) if every left $R$-module with property (I) (resp (S)) is Artinian (resp. Noetherian). It is known that a subring $B$ of a left I-ring (resp. S-ring) $R$ is not in general a left $\mathrm{I}$-ring (resp. S-ring) even if $R$ is a finitely generated $B$-module, for example the ring $M_{3}(K)$ of $3 \times 3$ matrices over a field $K$ is a left I-ring (resp S-ring), whereas its subring
\end{abstract}

$$
B=\left\{\left[\begin{array}{lll}
\alpha & 0 & 0 \\
\beta & \alpha & 0 \\
\gamma & 0 & \alpha
\end{array}\right] / \alpha, \beta, \gamma \in K\right\}
$$

which is a commutative ring with a non-principal Jacobson radical

$$
J=K \cdot\left[\begin{array}{lll}
0 & 0 & 0 \\
1 & 0 & 0 \\
0 & 0 & 0
\end{array}\right]+K \cdot\left[\begin{array}{lll}
0 & 0 & 0 \\
0 & 0 & 0 \\
1 & 0 & 0
\end{array}\right]
$$

is not an I-ring (resp. S-ring) (see [4], theorem 8). We recall that commutative I-rings (resp S-rings) are characterized as those whose modules are a direct sum of cyclic modules, these rings are exactly commutative, Artinian, principal ideal rings (see [1]). Some classes of non-commutative I-rings and Srings have been studied in [2] and [3]. A ring $R$ is of finite representation type if it is left and right Artinian and has (up to isomorphism) only a finite number of finitely generated indecomposable left modules. In the case of commutative rings or finite-dimensional algebras over an algebraically closed field, the classes of left I-rings, left S-rings and rings of finite representation type are identical (see [1] and [4]) A ring $R$ is said to be a ring with polynomial identity (P. I-ring) if there exists a polynomial $f\left(X_{1}, X_{2}, \ldots, X_{n}\right), n \geq 2$, in the non-commuting indeterminates $X_{1}, X_{2}, \ldots, X_{n}$ over the center $Z$ of $R$ such that one of the monomials of $f$ of highest total degree has coefficient 1 , and $f\left(a_{1}, a_{2}, \ldots, a_{n}\right)=0$ for all $a_{1}, a_{2}, \ldots, a_{n}$ in $R$. Throughout this paper all rings considered are associative rings with unity, and by a module $M$ over a ring $R$ we always understand a unitary left $R$-module. We use $M_{R}$ to emphasize that $M$ is a unitary right $R$-module.

KEY WORDS AND PHRASES: Left I-ring, left S-ring, ring with polynomial identity, ring of finite representation type.

1991 AMS SUBJECT CLASSIFICATION CODES: 16D70, 16P10, $16 \mathrm{~L} 60$. 


\section{THE MAIN RESULT}

THEOREM. Let $R$ be a left I-ring (resp. S-ring), and $B$ be a sub-ring of $R$ contained in the center $Z$ of $R$ Suppose that $R$ is a finitely generated flat $B$-module Then $B$ is an I-ring (resp S-ring)

To prove this theorem we need some results.

It is easy to see that

LEMMA 1. Every homomorphic image of a left I-ring (resp S-ring) is a left I-ring (resp S-ring)

LEMMA 2. Let $P_{1}$ and $P_{2}$ be two prime ideals of a ring $R$. If $P_{1}$ is not contained in $P_{2}$ then $\operatorname{Hom}_{R}\left(R / P_{1}, R / P_{2}\right)=\{0\}$

PROOF. Let $f: R / P_{1} \rightarrow R / P_{2}$ be an $R$-homomorphism, and set $f\left(1+P_{1}\right)=t+P_{2}$, where $t \in R$. Let $x \in P_{1} \backslash P_{2}$, and let $r$ be any element in $R$. We have $P_{2}=f\left(x r+P_{1}\right)=x r t+P_{2}$ Thus $x R t \in P_{2}$. Since $P_{2}$ is prime, we have $t \in P_{2}$, and hence $f=0$.

LEMMA 3. Let $R$ be a prime ring with polynomial identity. If $R$ is a left I-ring (resp. S-ring), then $R$ is simple Artinian.

PROOF. Let $R^{\prime}$ be the total ring of fractions of $R$ [5]. It is known that $R^{\prime}$ is simple Artinian [5], so the $R$-module $R^{\prime}$ satisfies (I) (resp. (S)). Since $R$ is a left I-ring (resp. S-ring), then $R^{\prime}$ is an Artinian (resp. Noetherian) $R$-module and hence $R^{\prime}=R$.

LEMMA 4. Let $R$ be a semi-prime ring with polynomial identity. If $R$ is a left I-ring (resp S-ring), then $R$ is semi-simple Artinian.

PROOF. Let $\left(P_{\ell}\right)_{\ell \in L}$ be a family pairwise distinct minimal prime ideals of $R$ such that

$$
\bigcap_{\ell \in L} P_{\ell}=\{0\} \text {. }
$$

By Lemma 1 the quotient rings $R / P_{\ell}(\ell \in L)$ are left I-rings (resp. S-rings) with polynomial identity Then it follows from Lemma 3 that the rings $R / P_{\ell}(\ell \in L)$ are simple Artinian, so the left $R$-modules $R / P_{\ell}(\ell \in L)$ satisfy (I) (resp (S)). Following Lemma $1, \operatorname{Hom}_{R}\left(R / P_{\ell}, R / P_{\ell}\right)=\{0\}$ for $\ell \neq \ell^{\prime}$, so the left $R$-module $M=\oplus_{\ell \in L} R / P_{\ell}$ satisfies (I) (resp. (S)). Since $R$ is a left I-ring (resp. S-ring), then $M$ is Artinian. But $R$ regarded as left $R$-module is isomorphic to a submodule of the semi-simple Artinian left $R$-module $M$, hence $R$ is semi-simple Artinian.

PROPOSITION 5. Let $R$ be a ring with polynomial identity. If $R$ is a left S-ring (resp. I-ring), then $R$ is left Artinian.

PROOF. Suppose that $R$ is a left S-ring (resp. I-ring) then the quotient ring $R / \operatorname{rad}(R)$, where $\operatorname{rad}(R)$ is the prime radical of $R$, is a left S-ring (resp. I-ring), so, following Lemma 4, the ring $R / \operatorname{rad}(R)$ is semi-simple Artinian This fact implies that $R$ is semi-perfect and hence $\operatorname{rad}(R)=J(R)$, where $J(R)$ is the Jacobson radical of $R$. Let $e$ be a primitive idempotent of $R$. Since the endomorphism ring of the $R$-module $R e$ is isomorphic to the local ring $e R e$ with a nil Jacobson radical $e J(R) e$, then the $R$-module $R e$ satisfies property (I) (resp (S)). It follows that the $R$-module $R e$ is Noetherian (resp. Artinian). Since $R$ regarded as $R$-module is a direct sum of finitely many left $R$-modules of the form $R e$, where $e$ is a primitive idempotent of $R$, then $R$ is Noetherian. Let $P$ now be a prime ideal of $R$. Since the prime ring $R / P$ is simple in virtue of Lemma 3, then $R$ is left Artinian.

PROOF OF THE MAIN THEOREM. Since $R$ is a finitely generated $Z$-module, then $R$ is a ring with polynomial identity (see [6]). So by Proposition $5 R$ is a left Artinian ring. Thus by [7] the ring $B$ is Artinian. Let $e_{1}, \ldots, e_{n}$ be primitive idempotents of $B$ such that $B=\oplus_{i=1}^{n} e_{2} B e i$ For every $i$, $1 \leq i \leq n, B_{2}=e_{2} B e_{2}$ is a local Artinian ring. To show that $B$ is a left I-ring (resp. S-ring) it is enough to show that for every $i, 1 \leq i \leq n, B_{\imath}$ is a left I-ring (resp. S-ring). We have $A=\oplus_{i=1}^{n} A_{i}$, where $A_{2}=e_{2} A e_{2}, 1 \leq i \leq n$. By hypothesis the left $B$-module $\oplus_{i=1}^{n} A_{2}=A$ is flat and finitely generated, so the $B_{i}$-module

$$
A_{2}=e_{2} A e_{\imath} \cong e_{i} A e_{2} \otimes_{B} B=A \otimes_{b} e_{2} B e_{2}=A \otimes_{B} B_{2}
$$


is also flat and finitely generated Since $B_{\imath}$ is an Artinian local ring then the $B_{\imath}$-module $A_{\imath}$ is faithfully flat (see [8] proposition 1, p. 44)

Suppose now that $B_{\imath}$ is not an I-ring (resp. S-ring) for some $i, 1 \leq i \leq n$ Then by Proposition 2 of [2], there exists a $B_{2}$-module $M$ of infinite length such that, for every integer $n \geq 1$, the $B_{1}$-module $M^{n}$ satisfies both properties (I) and (S) Following [8] (corollary 2, p. 107), the $B_{\imath}$-module $A_{\imath}$ is a free module. Let $M^{\prime}=M \otimes_{B_{1}} A_{2}$. Since the $B_{i}$-module $M$ is of infinite length and $A_{2}$ is a faithfully flat $B_{i}$-module, then $M^{\prime}$ is an $A_{2}$-module of infinite length. On the other hand, since $A_{\imath}$ is a free $B_{2}$-module, there exists an integer $s \geq 1$ such that $A_{2}=B_{2}^{s}$. We have then the $B_{\imath}$-module isomorphism

$$
M^{\prime}=M \otimes_{B_{1}} A_{2}=M \otimes_{B_{1}} B_{\imath}^{s} \cong M^{s} .
$$

Hence the $B_{2}$-module $M^{\prime} \cong M^{s}$ satisfies both properties (I) and (S) and therefore $M^{\prime}$, regarded as $A_{2}$-module, satisfies properties (I) and (S) This fact implies that the homomorphic image $A_{2}$ of the left Iring (resp. S-ring) $A$ is not a left I-ring (resp. S-ring), in contradiction with Lemma 1.

COROLLARY. Let $R$ be a left I-ring (resp. S-ring). If $R$ is a finitely generated flat module over its center $Z$, then $Z$ is an I-ring (resp. S-ring).

The following example shows that the converse of the theorem above is not true Let $K$ be a field The commutative ring $A=K[X, Y] /\left(X^{2}, X Y, Y^{2}\right)$ is not an I-ring (resp. S-ring) because its Jacobson radical $J=K \bar{X}+K \bar{Y}$ is not principal (see [1], theorem 8). On the other hand $K$ is an I-ring (resp $\mathrm{S}$-ring) and $A$ is a finite-dimensional $K$-vector space

ACKNOWLEDGEMENT. The author would like to thank the referee for his valuable suggestions and numerous very useful remarks about the text.

\section{REFERENCES}

[1] KAIDI, A.M. and SANGHARE, M., Une caractérisation des anneaux artiniens à idéaux principaux, Lec. Notes in Math., Vol. 1328, Springer-Verlag, Berlin (1988), 245-254.

[2] SANGHARE, M., Sur quelques classes d'anneaux liées au lemme de Fitting, Rend. Sem. Math. Padova, 87 (1992), 29-37.

[3] SANGHARE, M., On S-duo-rings, Comm. in Algebra 20 (8) (1992), 2183-2189

[4] SANGHARE, M., Characterizations of algebras whose modules with Fitting's property are of finite length, Ext. Mat. 7 (2) (1992), 1-2.

[5] POSNER, E.C., Prime rings satisfying a polynomial identity, Proc. Amer. Math. Soc. 11 (1960), 180-183.

[6] RENAULT, G., Algèbre non-commutative, Gauthier-Villars, Paris (1975).

[7] EISENBUD, E., Subrings of Artinian and Noetherian rings, Math. Ann. 185 (1970), 247-249

[8] BOURBAKI, N., Algèbre commutative chap. I, Hermann, Paris (1961). 


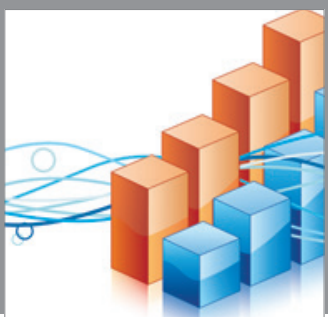

Advances in

Operations Research

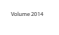

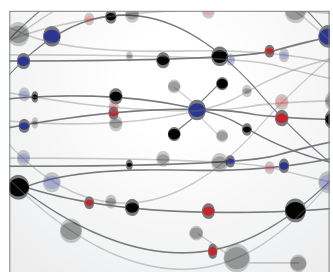

\section{The Scientific} World Journal
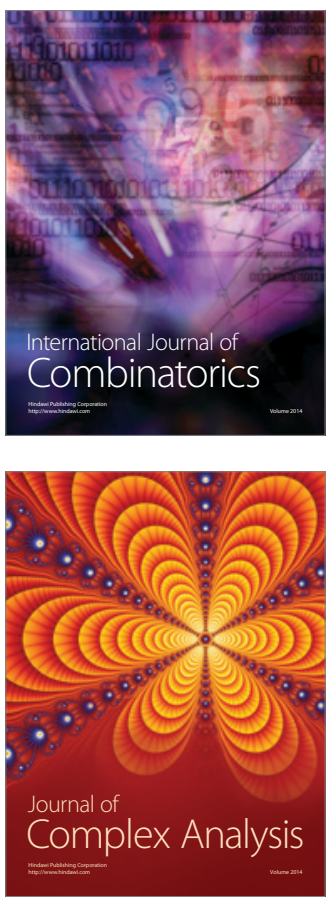

International Journal of

Mathematics and

Mathematical

Sciences
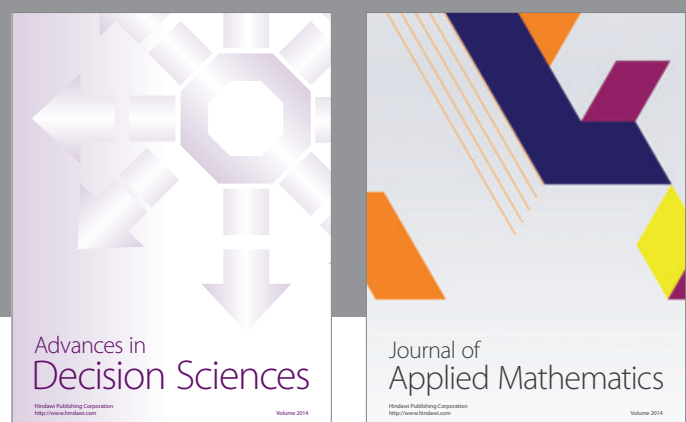

Journal of

Applied Mathematics
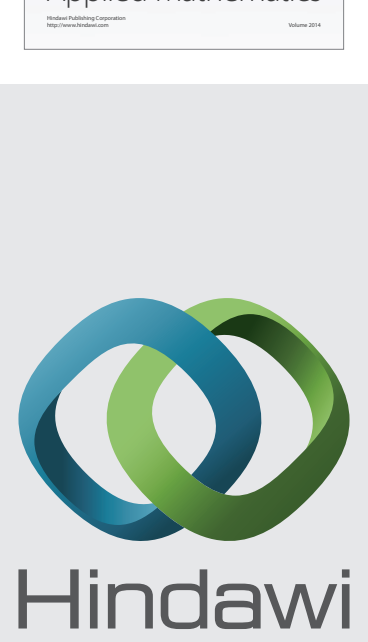

Submit your manuscripts at http://www.hindawi.com
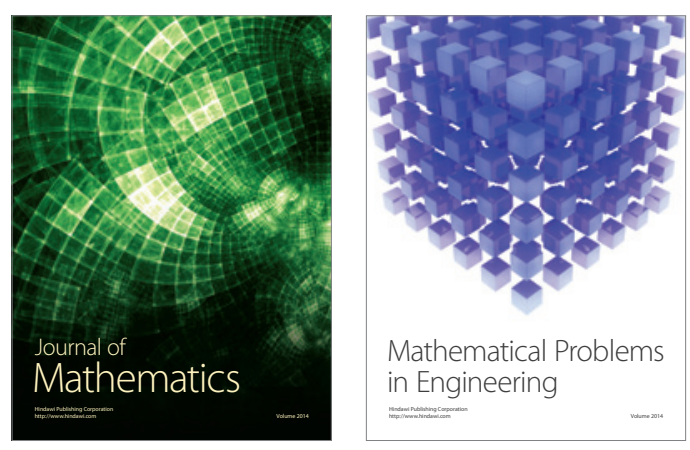

Mathematical Problems in Engineering
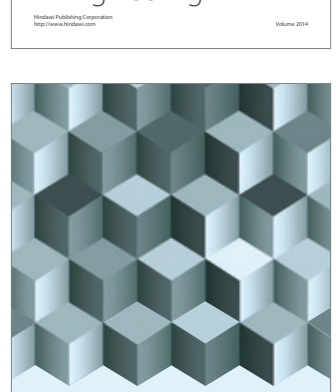

Journal of

Function Spaces
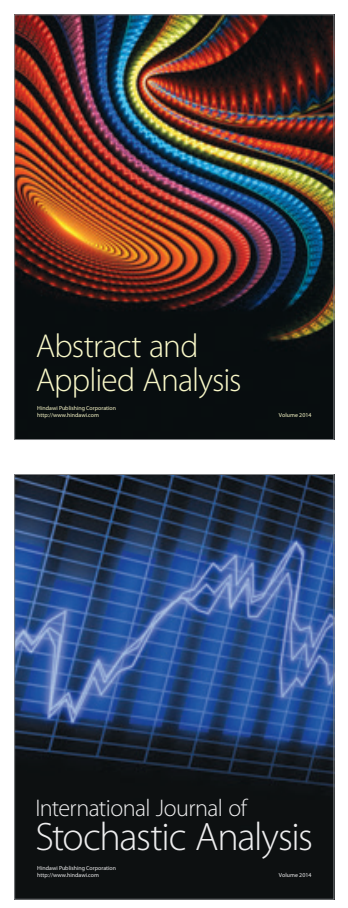

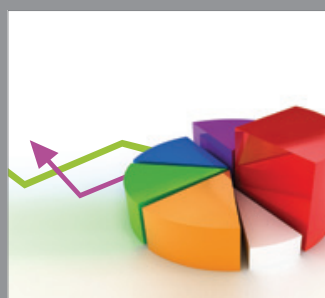

ournal of

Probability and Statistics

Promensencen
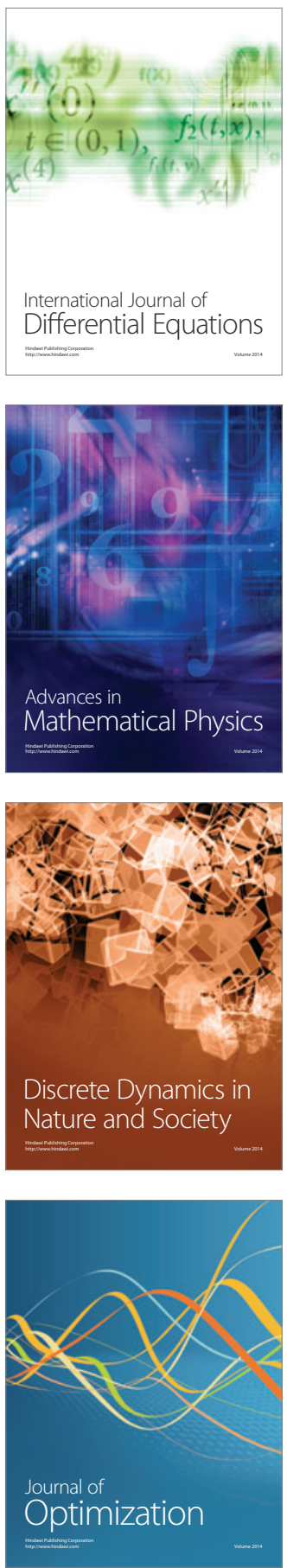